\title{
NITRIC OXIDE AND ALLYL ALCOHOL INDUCED HEPATOTOXICITY
}

\author{
I. YA. HORBACHEVSKY TERNOPIL STATE MEDICAL UNIVERSITY, TERNOPIL, \\ M. M. Korda
}

Background. Nitric oxide (NO) is an important mediator of hepatotoxicity. NO in liver can be derived from two sources: (1) constitutive NO synthase (eNOS) in endothelial cells, and (2) inducible NO synthase (iNOS) in hepatocytes and Kupffer cells.

Objectives. The present study was aimed to examine the effect of nonselective NOS inhibitor (L-NAME) and selective iNOS inhibitor (1400W) on the development of allyl alcohol (AA) induced hepatitis in rats.

Methods. Male Wistar rats were treated with intraperitoneal injection of saline or AA and L-NAME or 1400W. NO in liver was measured by electrochemical method after eNOS stimulation by calcium ionophore. Total NOS activity and nitrite/nitrate content were measured in liver and blood serum. The activity of free radical oxidation in liver was measured by chemiluminescent method. Alanine aminotransferase (ALT) and aspartate aminotransferase (AST) activities were assayed in blood serum

Results. AA increased the activity of free radical processes in liver and markers of cytolysis in serum, as well as decreased eNOS and increased iNOS activities. L-NAME considerably inhibited eNOS and augmented the necrosogenic properties of $A A$, whereas $1400 \mathrm{~W}$ partially prevented liver damage.

Conclusion. It has been concluded that in AA intoxication NO produced from eNOS is beneficial to the liver, but NO derived from the upregulated iNOS has deleterious effect.

KEY WORDS: nitric oxide, toxic hepatitis, NOS inhibitors.

\section{Introduction}

Allyl alcohol (AA) is a well known hepatotoxin widely used in animal experiments to induce liver necrosis. AA in hepatocytes is oxidized to acrolein by alcohol dehydrogenase [1]. Acrolein is a potent electrophile being able to attack nucleophilic molecules, e.g. sulfhydryl groups of reduced glutathione. The depletion of reduced glutathione affects the glutathione peroxidase antioxidant mechanisms and results in the activation of free radical oxidation. It has been suggested that the oxidative stress is the key event leading to liver necrosis in AA intoxication [2]. We used the AA model of hepatocyte cell damage in order to investigate the role of nitric oxide (NO) in the pathogenesis of necrotic liver injury. Nitric oxide (NO) is an important mediator of hepatotoxicity, and changes in its generation are paradoxically implicated in both cytoprotection and cytotoxicity [3]. NO in liver can be derived from two sources. Hepatocytes and Kupffer cells contain inducible NO synthase (iNOS), activity of which is markedly increased in inflammation. Endothelial cells contain constitutive NO synthase (eNOS). NO produced by eNOS plays important role in liver microcirculation. It has been shown that aminoguanidine, a relatively selective inhibitor of iNOS, protected against acetaminophen-

Address for correspondence: Mykhaylo Korda, Medical Biochemistry Department, I. Ya. Horbachevsky Ternopil State Medical University, m. Voli, 1, Ternopil, 46001, Ukraine Tel.: +380963952764; Fax: +380352253998;

E-mail:kordamm@yahoo.com induced hepatotoxicity [4], as well as thioacetamideinduced hepatotoxicity [5]. Another study concluded that iNOS plays an important role in liver cirrhosis following $\mathrm{CCl}_{4}$ intoxication to rats [6]. Recently, we have demonstrated that $\mathrm{N}$-(3-(Aminomethyl)benzyl)acetamidine (1400W), a strongly selective inhibitor of iNOS, prevented the depletion of reduced glutathione level in liver injured by allyl alcohol (AA) [7]. At the same time, in acute hepatitis caused by thioacetamide, methyl ester of N-nitro-L-arginine (L-NAME), the nonselective inhibitor of NOS, increased liver damage [8].

The present work was carried out to study the effects of nonselective (L-NAME) and selective iNOS $(1400 \mathrm{~W})$ inhibitors on the formation of $\mathrm{NO}$ in liver in AA intoxication, as well as to investigate the correlation between NO production and severity of the liver damage.

\section{Methods}

\section{Animals and treatment}

Male Wistar rats weighing 250-300 g were used in experiment. The animals were maintained under control conditions $\left(23^{\circ} \mathrm{C}, 12\right.$-hour light-dark cycle) with water and standard laboratory chow being available ad libitum. All rats were randomly assigned to four groups of ten animals each. Rats from the first group were treated with intraperitoneal (i.p.) injection of saline and served as a control. AA ( $30 \mathrm{mg} / \mathrm{kg}$ ) was diluted in saline and administered one time intraperitoneally into rats of the second, third and 
fourth groups. Third and fourth groups animals received correspondingly L-NAME (10 mg/kg, i.p.) and $1400 \mathrm{~W}$ (1.5 mg/kg, i.p.) $30 \mathrm{~min}$ prior to AA injection. On the next day of experiment rats were anesthetized with a mixture of $100 \mathrm{mg} / \mathrm{kg}$ ketamine and $10 \mathrm{mg} / \mathrm{kg}$ xylazine and liver was dissected to carry out the studies described below. Blood was also taken for the analysis.

All procedures were in compliance with national and international laws and guidelines for the use of animals in biomedical research [9].

\section{Measurement of NO in liver}

The measurement of NO was performed with highly sensitive electrochemical nanosensor which was prepared as previously described [10]. Briefly, a carbon fiber (tip diameter about $500 \mathrm{~nm}$ ) was covered with polymeric film of nickel (II) tetrakis (3-methoxy-4-hydroxy-phenyl) porphyrin. Measurements were performed using a three-electrode system that consisted of a nanosensor as the working electrode, a platinum counter electrode, and a silver-silver chloride reference electrode.

Liver tissue was cut into thin pieces of 5-6 mm in length using a razor blade and slices were placed on the bottom of thermostated organ chamber filled with Hank's solution. Counter and reference electrodes were positioned in the chamber near the liver slice and the active tip of the nanosensor was lowered near $(2-4 \mu \mathrm{m})$ the surface of liver tissue with the aid of micromanipulator.

To stimulate the release of NO, $10 \mu$ of eNOS receptor independent agonist calcium ionophore A23187 was injected with a nanoinjector onto the surface of liver slice to reach a final concentration in the medium of $1 \mu \mathrm{mol} / \mathrm{L}$.

The changes in NO concentration from the background level were monitored with time (amperometry) with a computer-based Gamry VFP600 multichannel potentiostat (detection limit of $1 \mathrm{nmol} /$ $\mathrm{L}$ and resolution time $<50 \mathrm{~ms}$ ). NO concentration was calculated by means of a calibration curve.

Measurement of the total NOS activity in liver and nitrite/nitrate content in liver and blood serum

The activity of $\mathrm{NO}$ generating system was assayed in $40 \mathrm{mM}$ Tris $\mathrm{HCl}$ buffer, $\mathrm{pH}$ 8, containing liver homogenate ( $1 \mathrm{mg}$ of protein/ml), $4 \mathrm{mM} \mathrm{FAD}$, $4 \mathrm{mM} \mathrm{H}_{4}$ biopterin, $3 \mathrm{mM}$ dithiothreitol, and $1 \mathrm{mM}$ $\mathrm{L}$-arginine. The reaction was initiated by adding $\mathrm{NADPH}(2 \mathrm{mM})$ and run for $3 \mathrm{~h}$ at $37^{\circ} \mathrm{C}$ [11]. The combined concentration of nitrite and nitrate, which accumulate quantitatively as the stable oxidation products of NO, was determined as described below.

Measurement of the nitrite/nitrate concentration

The combined nitrite/nitrate concentration in the NO generating system as well as in liver homogenate and blood serum was assayed after the reduction of nitrite to nitrate by cadmium using improved Griess method with the help of commercially available QuantiChrom $^{\mathrm{TM}}$ Nitric Oxide Assay Kit (BioAssay Systems).

\section{Free Radical Oxidation Assay}

The activity of free radical oxidation in liver homogenate was measured by chemiluminescent method [12].

\section{Evaluation of Hepatotoxicity}

Serum alanine aminotransferase (ALT) and aspartate aminotransferase (AST) activities were assayed as the markers of hepatocellular death using commercially available kits (Sigma-Aldrich).

\section{Statistical analysis}

Values are expressed as mean \pm SEM from 4 to 6 experiments. Values $\mathrm{P}<0.05$ are considered as statistically significant. Statistical analysis was performed using ANOVA followed by the Student $t$ test.

\section{Results}

The release of NO was measured ex-vivo using electrochemical nanosensors placed on the surface of the liver tissue. We stimulated NO release by a calcium ionophore, as it causes a large increase in intracellular calcium, rapid eNOS activation and maximal receptor-independent NO production. So far as, iNOS is not sensitive to $\mathrm{Ca}^{2+}$, we can assume that the amount of $\mathrm{NO}$ measured by this method directly reflects the functional state of the eNOS located in the hepatic sinusoidal endothelial cells. The maximum or peak concentration of released NO from the liver of control rats was $160.0 \pm 12.2 \mathrm{nmol} / \mathrm{L}$ (Figure 1). By contrast, stimulation of liver from the AA injured animals resulted in an approximately twofold reduction in the amount of NO release. Administration of the nonselective NOS inhibitor

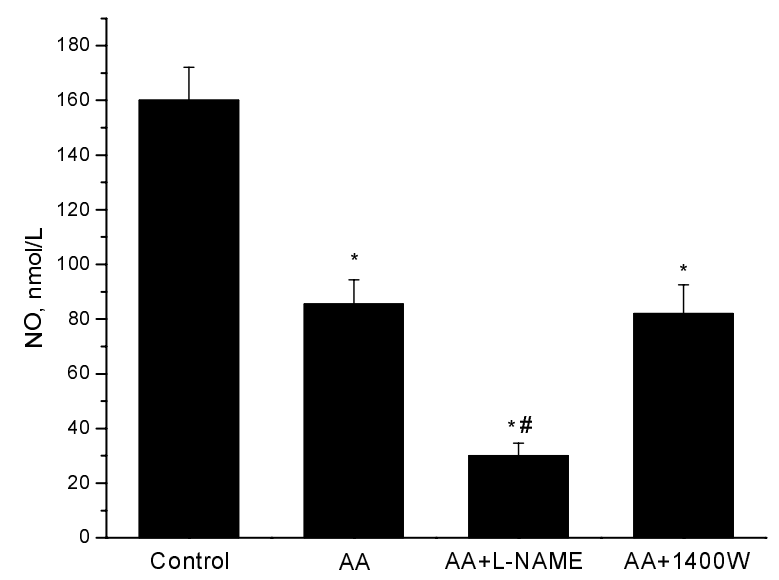

Fig. 1. The effect of L-NAME and $1400 \mathrm{~W}$ on calcium ionophore (A23187, $1 \mathrm{mmol} / \mathrm{L}$ ) stimulated nitric oxide release from liver of AA-treated rats. Rats were given AA (30 mg/kg, i.p.) with or without the administration of L-NAME (10 mg/kg, i.p.) or $1400 \mathrm{~W}(1.5 \mathrm{mg} / \mathrm{kg}$, i.p.) $30 \mathrm{~min}$ before AA. * - significantly different from control, $p<0.05$; - significantly different from AA alone, $p<0.05$. Values are mean \pm SEM of $5-6$ rats. 
L-NAME markedly decreased the peak NO release (more than in five times, as compared to control), whereas iNOS inhibitor $1400 \mathrm{~W}$ did not change the NO production significantly.

In contrast to the calcium ionophore stimulated $\mathrm{NO}$ yield, the activity of NO generating system was about 1.5-fold increased after intoxication, thereby suggesting that $A A$ liver damage is accompanied by the considerable iNOS induction (Figure 2). Both L-NAME and $1400 \mathrm{~W}$ significantly diminished the total NOS activity.

Serum and liver nitrite/nitrate concentrations correlated well with total NOS activity and were significantly increased 24 hours after AA admi-

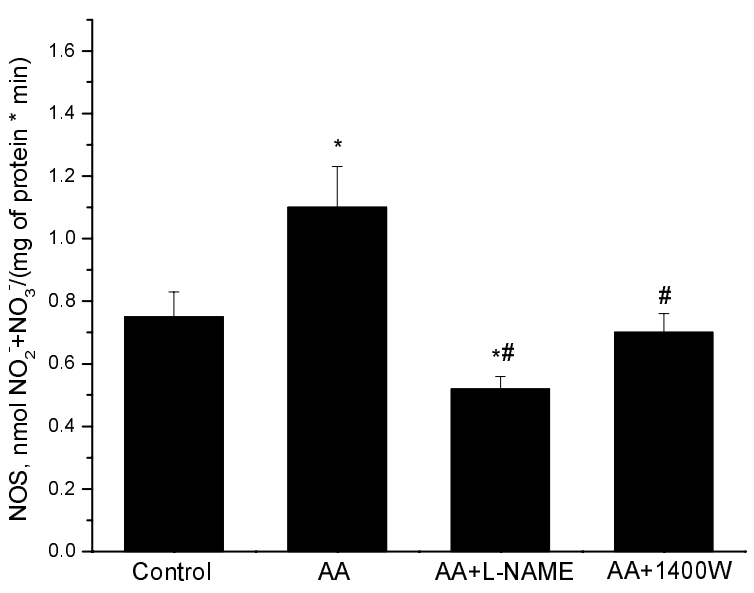

Fig. 2. The effect of L-NAME and $1400 \mathrm{~W}$ on the total NOS activity in liver of AA-treated rats. Rats were given AA ( $30 \mathrm{mg} / \mathrm{kg}$, i.p.) with or without the administration of L-NAME $(10 \mathrm{mg} / \mathrm{kg}$, i.p.) or $1400 \mathrm{~W}(1.5 \mathrm{mg} / \mathrm{kg}$, i.p.) $30 \mathrm{~min}$ before AA. * - significantly different from control, $\mathrm{p}<0.05$; \# - significantly different from AA alone, $p<0.05$. Values are mean \pm SEM of $6-8$ rats.

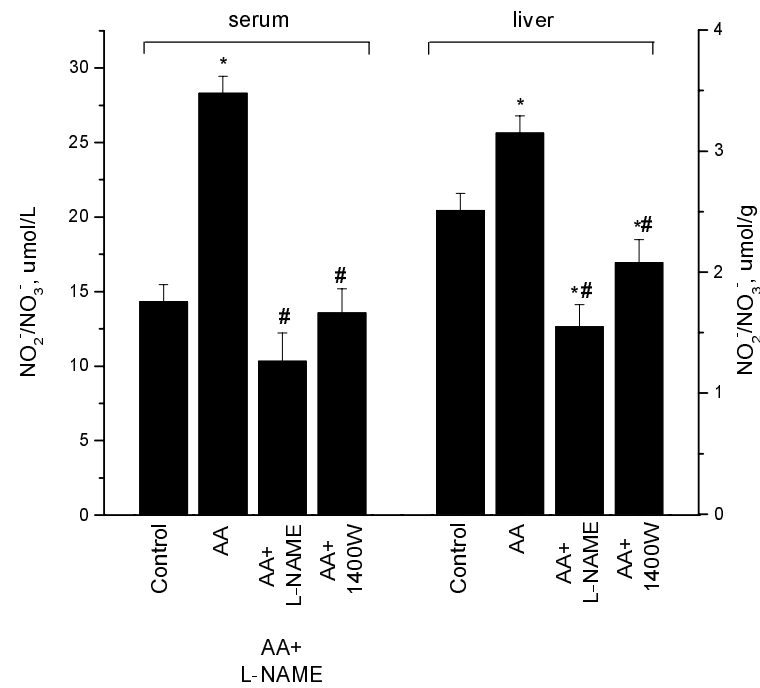

Fig. 3. The effect of L-NAME and $1400 \mathrm{~W}$ on the combined nitrite/nitrate content in blood serum and liver of AA-treated rats. Rats were given $A A(30 \mathrm{mg} / \mathrm{kg}$, i.p.) with or without the administration of L-NAME (10 mg/kg, i.p.) or $1400 \mathrm{~W}(1.5 \mathrm{mg} / \mathrm{kg}$, i.p.) $30 \mathrm{~min}$ before AA. * - significantly different from control, $\mathrm{p}<0.05$; \# - significantly different from AA alone, $p<0.05$. Values are mean \pm SEM of $6-8$ rats. nistration (Figure 3). Both inhibitors effectively prevented the increase in nitrite/nitrate level.

The activity of free radical oxidation evaluated by the intensity of spontaneous chemiluminescence of the liver homogenate was almost three-fold higher in the poisoned animals in comparison with control (Figure 4). It is worth noting that $1400 \mathrm{~W}$ significantly inhibited the free radical processes in hepatocytes, whereas L-NAME administration resulted in the increase of the chemiluminescence intensity.

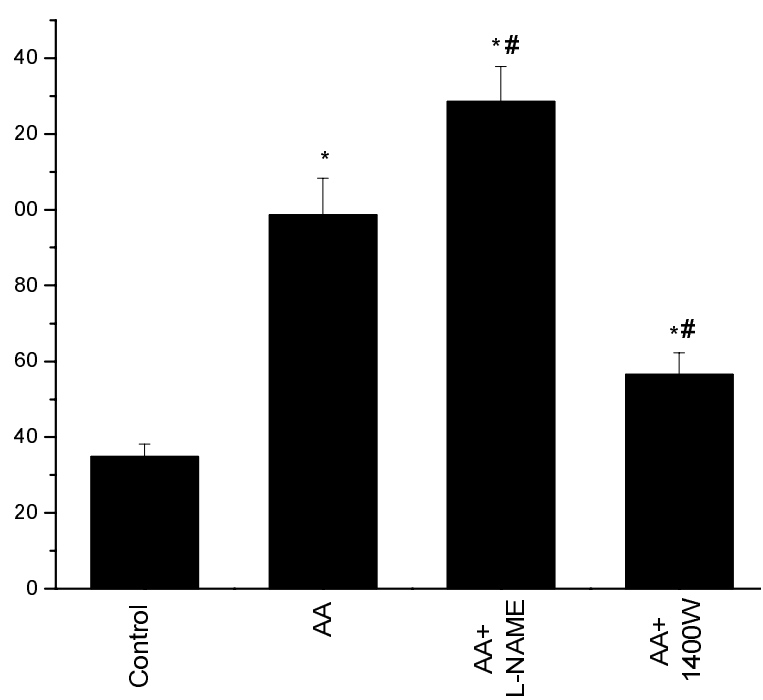

Fig. 4. The effect of L-NAME and $1400 \mathrm{~W}$ on the chemiluminescence intensity of AA-treated rats liver homogenate. Rats were given AA (30 mg/kg, i.p.) with or without the administration of L-NAME $(10 \mathrm{mg} / \mathrm{kg}$, i.p.) or $1400 \mathrm{~W}(1.5 \mathrm{mg} / \mathrm{kg}$, i.p.) $30 \mathrm{~min}$ before AA. * - significantly different from control, $p<0.05$; ${ }^{*}-$ significantly different from $A A$ alone, $p<0.05$. Values are mean \pm SEM of $6-8$ rats.

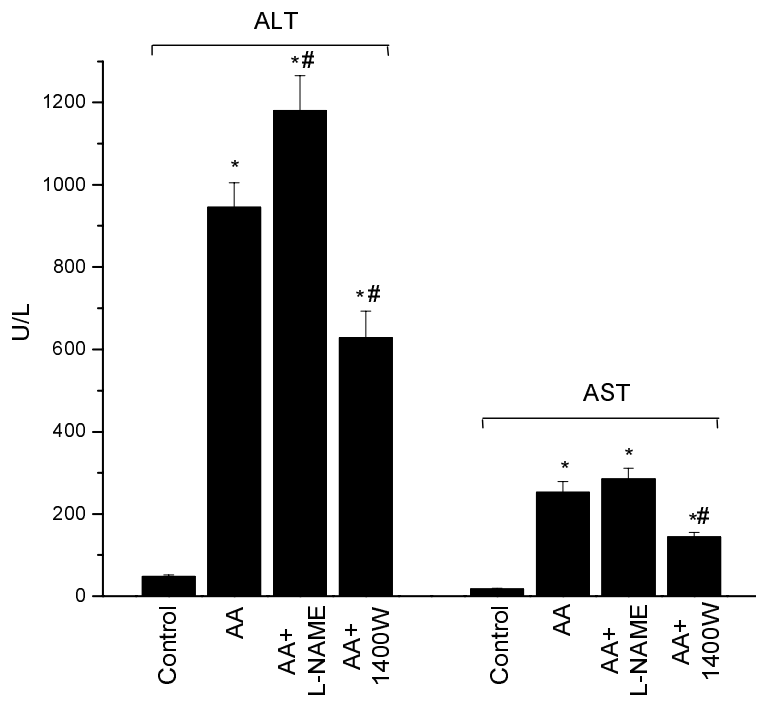

Fig. 5. The effect of L-NAME and 1400W on ALT and AST activities in blood serum of AA-treated rats. Rats were given AA $(30 \mathrm{mg} / \mathrm{kg}$, i.p.) with or without the administration of L-NAME $(10 \mathrm{mg} / \mathrm{kg}$, i.p.) or $1400 \mathrm{~W}(1.5 \mathrm{mg} / \mathrm{kg}$, i.p.) $30 \mathrm{~min}$ before $A A .{ }^{*}-$ significantly different from control, $p<0.05$; \#- significantly different from $A A$ alone, $p<0.05$. Values are mean \pm SEM of $6-8$ rats. 
The activities of serum ALT and AST indicate the presence of hepatocyte cytolysis in animals treated with AA. These markers were especially elevated in the group administered with $A A$ and L-NAME, which suggests significant damage of hepatocyte membranes in these rats (Figure 5). iNOS inhibitor partially decreased ALT and AST activity, as compared to the animals treated with AA alone.

\section{Discussion}

It is generally accepted that NO is an important regulator in the hepatotoxicity of many chemicals [4, $5,6]$. However, the role of NO in the development of liver necrosis is still not fully known and demands further studies. NO is reported to be produced in liver by both eNOS (endothelial cells) and iNOS (parenchymal and Kupffer cells). Previously we have shown that necrosogenic poison AA sharply decreases the reduced glutathione content in hepatocytes but inhibition of iNOS partially prevents this effect [7]. In this study we demonstrate thatAA rapidly upregulates iNOS in liver. Depletion of the glutathione may weaken cellular antioxidant defense to such a point that the NO produced by iNOS may cause tissue injury. This suggestion is confirmed by our observation. The activity of iNOS as well as the intensity of free radical processes in liver tissue and the markers of cytolysis in serum (ALT and AST), which were markedly

\section{References}

1. Belinsky SA, Bradford BU, Forman DT, Glassman EB, Felder MR, Thurman RG. Hepatotoxicity due to allyl alcohol in deermice depends on alcohol dehydrogenase. Hepatology 1985; 5: 1179-1182.

2. Pompella A, Romani A, Benedetti A, Comporti M. Loss of membrane protein thiols and lipid peroxidation in allyl alcohol hepatotoxicity. Biochem Pharmacol 1991; 41: 1255-1259.

3. Wang Y, Vodovotz Y, Kim PK, Zamora R, Billiar TR. Mechanisms of hepatoprotection by nitric oxide. Ann N Y Acad Sci 2002; 962: 415-422.

4. Gardner CR, Heck DE, Yang CS, Thomas PE, Zhang XJ, Degeorge GL, Laskin JD, Laskin DL. Role of nitric oxide in acetaminophen-induced hepatotoxicity in rat. Hepatology 1998; 27: 748-754.

5. Diez-Fernandez C, Sanz N, Alvarez AM, Zaragoza A, Cascales M. Influence of aminoguanidine on parameters of liver injury and regenaration induced in rats by a necrogenic dose of thioacetamide. $\mathrm{Br} \mathrm{J}$ Pharmacol 1998; 125: 102-108.

6. Ortiz MC, Fortepiani LA, Martinez C, Atucha NM, Gracia-Estan J. Renal and pressure effects of amino- increased after AA injection, went down significantly in animals treated with the strongly selective iNOS inhibitor $1400 \mathrm{~W}$. These results distinctly show that the upregulation of iNOS in macrophages and hepatocytes following by the activation of free radical reactions in liver cells may be at least one of the AA necrosogenic effect mechanisms.

We also demonstrate here that in contrast to iNOS, hepatic eNOS is significantly inhibited in liver necrosis. This effect of AA, like the activation of iNOS, can also be the reason of the necrotic liver damage. After administration of the nonselective NOS inhibitor L-NAME we observed the deep depression of eNOS activity simultaneously with the considerable raise of the chemiluminescence intensity in liver and cytolytic markers activity in serum. This phenomenon can be be explained by the role of NO derived from eNOS in maintaining perfusion, and preventing hypoxia, platelet adhesion and thrombosis in liver capillaries.

\section{Conclusion}

In the AA intoxication NO produced from eNOS is clearly beneficial to the liver, whereas the inducible NO production has an opposite effects. Further research should provide a solid basis for therapeutic approaches to either supplement NO to the liver for its protective effect or suppress iNOS to prevent liver damage.

guanidine in cirrhotic rats with ascites. J Am Soc Nephrol 1996; 7: 2694-2699.

7. Korda MM, Yaroshenko TYa. The effect of iNOS inhibitor $\mathrm{N}$-(3-(Aminomethyl)benzyl) acetamidine on the hepatotoxicity of allyl alcohol. Med Chem 2004; 6: 114-117.

8. Chu CJ, Wang SS, Lee FY, Chang FY, Lin HC, Hou MC, Chan CC. Detrimental effects of nitric oxide inhibition on hepatic encephalopathy in rats with thioacetamide-induced hepatic failure. Eur J Clin Invest 2001; 31: 156-163.

9. Giles AR. Guidelines for the use of animals in biomedical research. Thromb Res 1987; 58: 1078-1084.

10. Malinski T, Taha Z. Nitric oxide release from a single cell measured in situ by a porphyrinic based microsensor. Nature 1992; 358: 676-678.

11. Stuehr D, Kwon NS, Nathan C, Griffiths O. $\mathrm{Nw}$-Hydroxy-L-arginine is an intermediate in the biosynthesis of nitric oxide fromL-arginine. J Biol Chem 1991; 266: 6259-6263.

12. Korda MM. Lipid peroxidation is a prerequisite for galactosamine-induced damage. Curr. Topics in Biophys 2000; 24(2): 83-87.

Received: 2014.06.01 